\title{
Analyzing the performance of low stage interconnection network
}

\begin{abstract}
In order to avoid crosstalk, a new architecture is proposed for Optical Multistage Interconnection Networks (OMINs). In the new architecture, two switches are replaced by one switch in each row. Reduction in the number of switches makes the considerable reduction in the execution time. To study the performance of the new architecture, analytical techniques also can be used effectively. The theory of probability is used to derive mathematical equation for network bandwidth allocation of a unit load. The obtained results show the improvement in the network performance. By increasing load, the bandwidth is reduced. In addition the simulation is applied to validate the new architecture and show improvement in the performance by approximately $30 \%$ reduction in the execution time.
\end{abstract}

Keyword: Bandwidth; Crosstalk; Low stage interconnection network; Switch element 